\title{
COUPLED ANALYTICAL AND NUMERICAL APPROACH TO UNCOVERING NEW REGULATORY MECHANISMS OF INTRACELLULAR PROCESSES
}

\author{
JAROSŁAW ŚMIEJA \\ Institute of Automatic Control \\ Silesian University of Technology, ul. Akademicka 16, 44-100 Gliwice, Poland \\ e-mail:Jaroslaw.Smieja@polsl.pl
}

\begin{abstract}
The paper deals with the analysis of signaling pathways aimed at uncovering new regulatory processes regulating cell responses. First, general issues of comparing simulation and experimental data are discussed, and various aspects of data normalization are covered. Then, a model of a particular signaling pathway, induced by Interferon- $\beta$, is briefly introduced. It serves as an example illustrating how mathematical modeling can be used for inferring the structure of a regulatory system governing the dynamics of intracellular processes. In this pathway, experimental results suggest that a hitherto unknown process is responsible for a decrease in the levels of one of the important molecules used in the pathway. Then, equilibrium points of the model are analyzed, allowing the rejection of all but one explanation of the phenomena observed experimentally. Numerical simulations confirm that the model can mimic the dynamics of the processes in the pathway under consideration. Finally, some remarks about the applicability of the method based on an analysis of equilibrium points are made.
\end{abstract}

Keywords: signaling pathways, equilibrium points, simulation.

\section{Introduction}

Recent advances in experimental procedures used to gather biological data have led to much better understanding of the nature of intracellular processes. Though our knowledge of these processes has rapidly been expanded, still much more remains to be uncovered. Researchers' efforts are hampered by at least several factors, high costs of experiments being not the least of them.

Various approaches to mathematical modeling of biological processes have been developed. Capturing the dynamics of these processes through mathematical analysis can facilitate further experimental developments. However, many more models have been developed for the analysis of population dynamics than for complex intracellular processes. One of the reasons for that is the lack of precise knowledge about regulatory mechanisms governing those processes. However, it is mathematical and/or numerical analysis that provides tools for testing hypothetical regulatory mechanisms before devoting significant resources for experimental work.

There are various ways in which signaling pathways can be modeled, depending on the goal of modeling. In this paper, a deterministic approach is used, in which the dynamics of intracellular processes are described by me- ans of ordinary differential equations.

Contrary to standard approaches for the identification of processes and their parameters, used, e.g., in control theory and its applications, models of pathways cannot be built as input-output ones. They must relate directly to biochemical processes involved in regulatory networks. There are two main reasons for such an approach. First, to uncover new mechanisms regulating intracellular processes, they must be explicitly described. Second, these mechanisms vary from one cell type to another (Fig. 1). If one chose an input-output representation, it had to be built from scratch for each cell type and it would be impossible to use knowledge gained in other experiments.

Before any conclusions are drawn, however, one should be aware of experimental procedures used in molecular biology and their limitations. The first, and one of the most important questions to be asked, is if the experimental results represent the dynamics of processes in individual cells, or average population behavior. If the latter is the answer, then these results should be handled very carefully. The heterogeneity of cells might lead to false conclusions about the nature of time responses in the analyzed systems (see Fig. 2). Although the analysis of equilibrium points, e.g., by means of Lyapunov theory, can provide us 


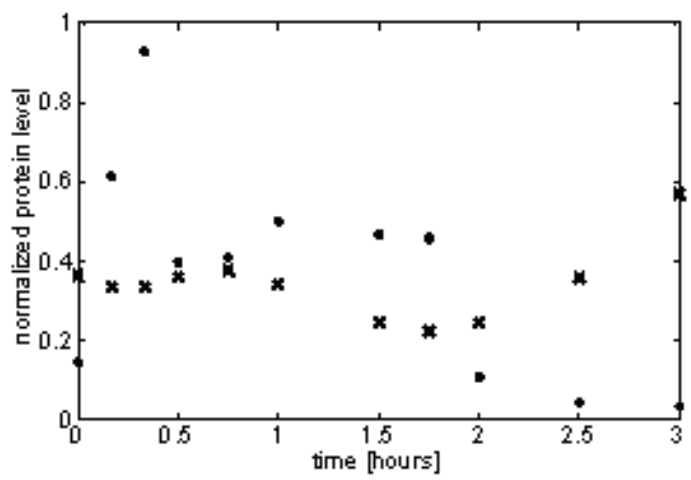

Fig. 1. Exemplary experimental results showing levels of a cytoplasmic IRF1 protein in HeLa cells (circles) and A549 cells (crosses), illustrating different regulatory mechanisms involved in controlling this protein in different cell types.

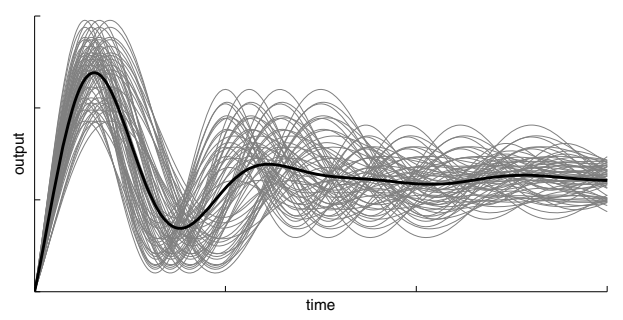

(a)

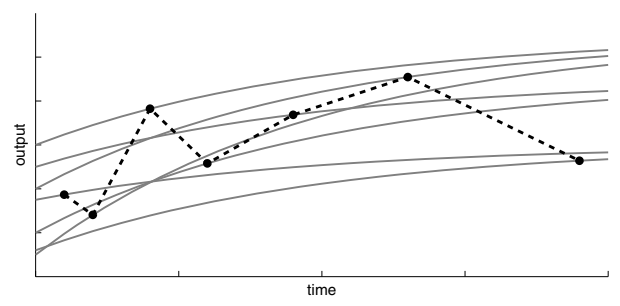

(b)

Fig. 2. Dynamical artifacts in experimental data, when the procedure does not trace the behavior of individual cells: actual oscillations in individual cells are cancelled on the population level (a); misleading oscillations observed when the replicate cell plates have been used in the order shown by numbers in the plot (b).

with information about their character, before comparing simulation results to experimental data, we must make sure that the latter represent real dynamics. Only then will the investigation of the pathway presented in subsequent sections yield meaningful results.

The general concept of deterministic modelling and fitting model parameters into data coming from Western Blots, EMSA blots, Real-Time PCR, and so forth is based on the notion of the homogeneity of the cell population used in experiments. In fact, even if all cells are cloned from the same ancestor, they are not homogenous in the sense of the dynamics of intracellular processes. The heterogeneity can be attributed to stochastic distribution of initial conditions (e.g., interpreted as the number of molecules of a given type at time 0 ) or differences in kinetic parameters of analyzed processes. As a result, different dynamics of intracellular processes may be observed. This can lead to deceptive artifacts, considering that experimental procedures consist in growing cell cultures on different plates, each of which is subsequently used to get measurement for one time point (see Fig. 2 (b)). Due to differences in initial conditions and kinetic constants for different cells (even of the same type), such procedures can lead to quantitatively (but not qualitatively) different responses in cell subpopulations, grown on a replicate plates one for each time point measurement. The character of the genuine system response can be distorted falsely implying oscillatory behaviour. A clear experimental example is shown in Fig. 7, where initial, highly variable levels of molecules most likely do not represent genuine oscillations in the system (which was confirmed by other experiments). The most likely explanation of the observed phenomenon is the variability in dynamics between replicate cell plates.

The even more important aspect of heterogeneity of the cell population was analyzed by Lipniacki et al. (2006). If there are large oscillations in single cells, data gathered during the experiments do not reflect real dynamics at all and therefore cannot be used for parameter fitting. When cells are exposed to an external excitation, the initial response can be relatively well synchronized. However, in the course of the experiment, cells lose their initial synchronicity, and the actual oscillations characteristic for the dynamics of a given pathway are lost through the averaging nature of the experiment (Fig. 2(a)). Once again, as in the preceding subsection, the problem lies in the heterogeneity of the cell population. As a consequence, single cell experiments are then required. Moreover, in such cases it does not make any sense to build models based on averaging experimental techniques.

\section{Normalization of results}

Models of signaling pathways most often use the molar concentration of molecules, or the average number of molecules of a given type as state variables. However, experimental procedures offer only relative results obtained from measurements. There exist virtually no databases containing information about the actual content of cells in terms of typical concentrations of known proteins, complexes or other molecules. Though it is theoretically possible to perform experiments that would provide such data, the cost involved is too high and, as a result, in most cases available data allow comparing only the levels of a given molecule to the initial level of molecules of the same ty- 


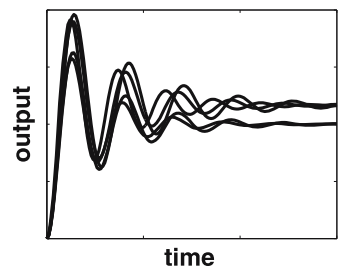

a)

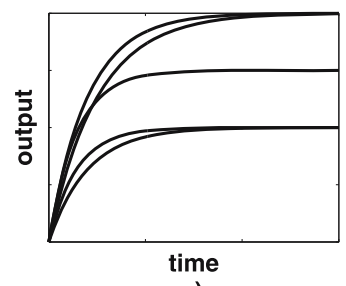

c)

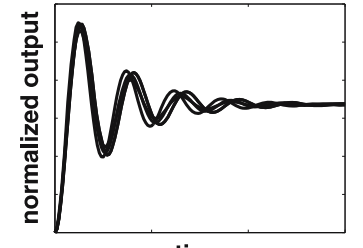

b)

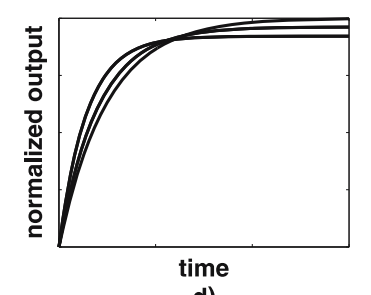

d)
Fig. 3. Effect of normalization on results comparison: when normalized to the area under the plot, most of the variation disappears: original (a) and normalized oscillatory response (b), and original (c) and normalized (d) aperiodic response.

pe. Therefore, to allow a comparison of experimental data coming from different sources and simulation results, the normalization of the results, both experimental and numerical, is necessary.

Two most common approaches consist in normalization either to the maximum value or to the area under the plot representing the time course of a given variable. If the latter is used, it is possible to minimize the influence of distorted measurements. However, this also restricts research to qualitative analysis only, as systems exhibiting the same type of dynamics, but differing in parameters, after normalization can even be indistinguishable (Fig. 3). Therefore, one should also check original results (before normalization) in order to form more sophisticated hypotheses.

It should be noted, however, that if experimental data come from different sources, usually the time horizon for each set of data is different. Then, either only partial results can be taken into account, or other normalization procedures should be considered (see Fig. (4).

It is a standard approach in the analysis of dynamical systems to search for the best models in an automated way, whenever possible, usually minimizing a square error between the simulation and experimental results, calculated at discrete time points. However, taking into account phenomena described in preceding subsections, one should allow for relatively large values of such an error, due to the uncertainty of measurements. Then, fully automatic procedures could lead to false conclusions about the dynamics of investigated processes (Fig. 5). This is particularly important in view of a small number of time points taken into account in experiments.

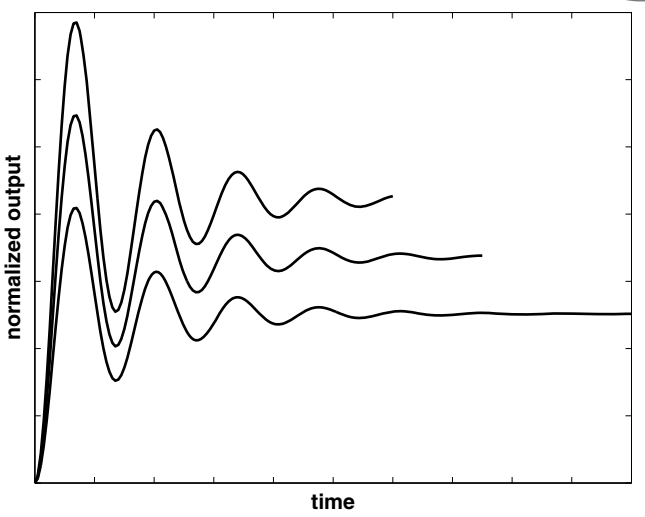

Fig. 4. Comparing results normalized to the area under the plot when different time scales are involved: although all plots represent the same function, they look different.

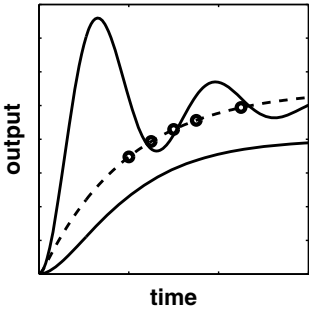

(a)

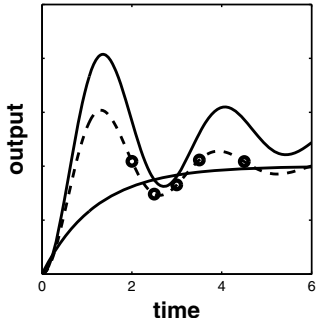

(b)
Fig. 5. Two cases, when instead of dynamics that are quantitatively similar to the actual ones (shown using the dotted line) the other could be chosen due to a smaller square error calculated at points shown by circles.

Despite all the obstacles, some of which are listed above, mathematical models can provide valuable insight into the mechanisms regulating cell responses on the intracellular level. Subsequent sections show how initial and late cell responses can be analyzed in a particular pathway.

\section{Rough introduction into the Interferon- $\beta$ signaling pathway}

Interferons (IFNs) are very important components of the immunodefense system (Janeway, 2001; Bekisz et al., 2004; Pestka et al., 2004). Their role and elements of interferon-induced signaling pathways are subjects of ongoing research (see, e.g., the reviews by Sen (2001), Levy and Darnell (2002), Kalvakolanu (2003) or Shuai and Liu (2003)).

The graphical representation of the pathway is presented in Fig. 6, The most important molecules mediating cell responses after IFN (both type I and II) stimulation are STAT (Signal Transducer and Activator of Transcription) proteins. In particular, two members of this family of proteins, STAT1 and STAT2, mediate the responses taken 


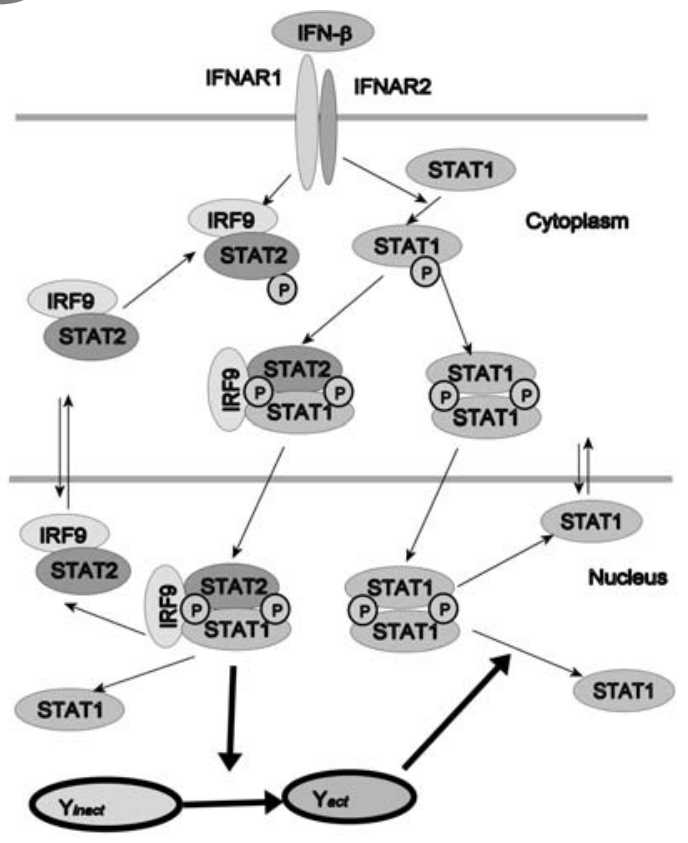

Fig. 6. Early processes in the Interferon- $\beta$ activated pathway.

into account in the analyzed pathway. Binding of IFN- $\beta$ to a cell receptor results in the phosphorylation of STAT proteins. The intermediate stages of this process are not directly modeled. Instead, the process is assumed to follow simple first-order dynamics. Subsequently, phosphorylated STATs form hetero- and homodimers. In the cytoplasm, STAT1-STAT2 heterodimers form a complex with an IRF9 protein, called ISGF3. Both STAT1 homodimers and the ISGF3 complex are very rapidly transported into the nucleus, where they serve as active transcription factors of, among others, IRF1 and IRF7 genes, respectively. Phosphorylated STATs are dephosphorylated by phosphatases both in the nucleus and in the cytoplasm. Dephosphorylation results in the dissociation of complexes leading to nuclear export of STATs and making them available to subsequent phosphorylation/dephosphorylation cycles.

Newly produced IRF1 mRNA is translated into an IRF1 protein, which in turn is a transcription factor for the STAT1 gene. In this way, a positive feedback loop is created in this signaling pathway. Other positive feedback loops also exist (acting through, e.g, an IRF7 protein), but they are activated in the presence of a viral infection and therefore are not taken into account here.

The original model of an IFN- $\beta$ stimulated pathway was developed and presented by Śmieja et al. (2008). It provided a good fit to experimental data. There, a new process that regulates the homodimer level in the nucleus was postulated (Fig. 8). However, one could argue that the justification for the hypothesis about activated phosphatases is far fetched and that alternative explanations, if paired with appropriate kinetic constants in corresponding models, could be accepted. Moreover, the model failed to explain the source of cytoplasmic IRF1 accumulation, observed in the experiments, which took place about 1.5 to 2 hours after reaching its peak value in the nucleus (see Fig. 7). None of the known negative feedbacks could explain this phenomenon. In the following sections we show that the analysis of equilibrium points in the model can confirm the hypothesis stated by Śmieja et al. (2008), and numerical simulations provide a testable hypothesis about the other unknown regulatory process.

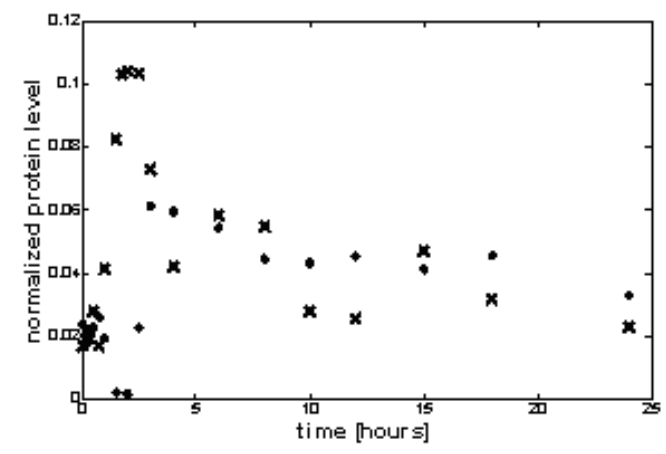

Fig. 7. Comparison of cytoplasmic (circles) and nuclear (crosses) levels of an IRF1 protein in Hela cells treated with IFN- $\beta$.

\section{Early stages of the Interferon- $\beta$ activated pathway}

The dynamics of the STAT1 homodimer nuclear level clearly show that there must be a regulatory mechanism responsible for its decrease after reaching a peak value after approximately 90 minutes of IFN treatment (see Fig. 8). Though several negative feedbacks in this pathway are widely known (see, e.g.,the findings of Alexander and Hilton (2004) or Wormald and Hilton (2004)), they cannot explain such a sharp decrease, as proved by numerical simulations and analytical deliberations further in this section.

In the work of Śmieja et al. (2008) it was postulated that the control mechanisms rely on increased dephosphorylation of homodimers in the nucleus. It was justified by numerical simulations that were run for various sets of parameters. In this section, the analysis concentrates on equilibrium points of the model, without making any assumptions about parameter values.

Two possible scenarios are taken into account. In the first one, the rate of homodimer degradation increases, while the second one consists in an increased rate of homodimer dephosphorylation.

The general form of models that are introduced in this section is based on the results of Śmieja et al. (2008). 


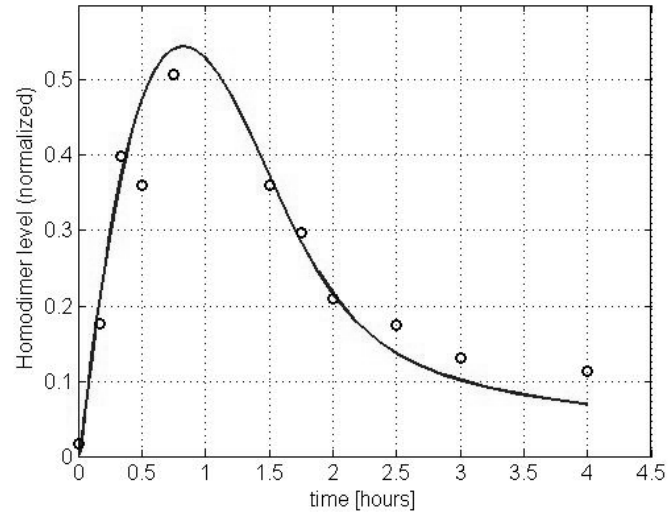

Fig. 8. Time course of homodimers of phosphorylated STAT1. Experimental data have been normalized to the area under the plot.

However, they need to be simplified if the analysis is to concentrate on equilibrium points in the most general case, when no assumptions about parameter values are made. The underlying idea of the simplification is that the process regulating homodimer behavior is too fast to rely on the synthesis of new mRNA molecules. Therefore, the process should be based on molecules existing in the cytoplasm and/or nucleus cell during the initial phase of the cell response to IFN. If so, then it would work also in cells in which the IRF1 gene is switched off. This yields the following simplifications in the original model:

- Much fewer variables are taken into account, since some processes rely on IRF1 produced in the initial phase of cell response. This includes both IRF1 mRNA, an IRF1 protein, and transcripts, whose transcription factor is an IRF1 protein or its complex.

- Since the STAT1 gene belongs to those whose transcription is activated by IRF1, only its constitutive transcription should be taken into account. As a result, only a constant production of an STAT1 protein is to be included in the model, and STAT1 mRNA can be neglected.

- It results from the previous assumption that the positive feedback in STAT1 production is broken in cells with the IRF1 gene switched off. Therefore, there will be no saturation in the phosphorylation rate for STAT1.

- The dephosphorylation of dimers in the cytoplasm is neglected, as they are very efficiently transported into the nucleus; the dynamics of this transport are also neglected.

- According to the experimental results, much more phosphorylated STAT2 is in the ISGF3 complex than in a heterodimer with phosphorylated STAT2. Therefore, heterodimers are neglected in the model.
- The system that will be investigated is under constant IFN treatment. This means that IFN is no longer an input. Instead, it is hidden in the phosphorylation kinetic rate as a constant parameter.

Then, if both possible hypotheses about additional regulatory mechanisms are incorporated in the model, the pathway dynamics are described by the following equations (the variable meaning is given in Table 1):

$$
\begin{aligned}
& \frac{\mathrm{d}(S 1)}{\mathrm{d} t}=k_{s 1 \_p r o d}-k_{s 1 \_d e g} \cdot(S 1)-i_{s 1} \cdot \\
& +e_{s 1} \cdot(S 1)_{n}-k_{s 1 \_p h o s} \\
& +k_{s 1_{-} d e p h} \cdot\left(S 1_{p}\right) \text {, } \\
& \frac{\mathrm{d}(S 2)}{\mathrm{d} t}=k_{s 1 \_p r o d}-k_{s 1 \_d e g} \cdot(S 1)-i_{s 2} \\
& +e_{s 2} \cdot(S 2)_{n}-k_{s 2 \_p h o s} \\
& +k_{s 2 \_d e p h} \cdot S 2_{p} \text {, } \\
& \frac{\mathrm{d}\left(S 1_{p}\right)}{\mathrm{d} t}=k_{s 1 \_p h o s} \cdot(S 1)-k_{s 1_{-} \text {deph }} \cdot\left(S 1_{p}\right) \\
& -2 k_{s 1 s 1} \cdot\left(S 1_{p}\right)^{2} \\
& -k_{S 12} \cdot\left(S 1_{p}\right) \cdot\left(S 2_{p}\right) \text {, } \\
& \frac{\mathrm{d}\left(S 2_{p}\right)}{\mathrm{d} t}=k_{s 2 \_p h o s} \cdot(S 2)-k_{s 2 \_d e p h} \cdot\left(S 2_{p}\right) \\
& -k_{S 12} \cdot\left(S 1_{p}\right) \cdot\left(S 2_{p}\right) \\
& \frac{\mathrm{d}(S 1)_{n}}{\mathrm{~d} t}=k_{v} i_{s 1} \cdot(S 1)-k_{v} e_{s 1} \cdot(S 1)_{n} \\
& +2 k_{i n v \_s 1 s 1 \_n} \cdot(S 11)_{n} \\
& +k_{\text {inv } S 12} \cdot(S 12)_{n} \\
& +2 k_{d x} \cdot(S 11)_{n} \cdot\left(X_{a c t}\right) \\
& \frac{\mathrm{d}(S 2)_{n}}{\mathrm{~d} t}=k_{v} i_{s 2} \cdot(S 2)-k_{v} e_{s 2} \cdot(S 2)_{n} \\
& +k_{\text {inv } 12} \cdot(S 12) \\
& \frac{\mathrm{d}(S 12)_{n}}{\mathrm{~d} t}=k_{v} k_{S 12} \cdot\left(S 1_{p}\right) \cdot\left(S 2_{p}\right) \\
& -k_{i n v S 12} \cdot(S 12)_{n} \text {, } \\
& \frac{\mathrm{d}(S 11)_{n}}{\mathrm{~d} t}=k_{v} k_{s 1 s 1} \cdot\left(S 1_{p}\right)^{2}-k_{i n v_{-} s 1 s 1} \cdot(S 11)_{n} \\
& -k_{d x} \cdot(S 11)_{n} \cdot\left(X_{a c t}\right) \\
& -k_{d y} \cdot(S 11)_{n} \cdot\left(Y_{a c t}\right) \text {. }
\end{aligned}
$$

All other symbols not mentioned above are model parameters, with $k_{v}$ representing the cytoplasmic-to-nuclear volume ratio, as in the work of Ciliberto et al. (2005).

In the last equation, the last line contains terms representing two possible hypotheses that could explain the dynamics of the nuclear homodimer level, observed in experiments. First of them corresponds to an increased dephosphorylation rate, while the second term represents induced degradation of homodimers. Both require the introduction of unknown molecules whose binding to homodimer results in a desired end effect. It will be assumed, as in 
Table 1. Proteins and corresponding variable names.

\begin{tabular}{|c|c|c|}
\hline Protein or complex & location & variable name \\
\hline \hline STAT1 & cytoplasm & $(S 1)$ \\
\hline STAT2 & cytoplasm & $(S 2)$ \\
\hline STAT1 & nucleus & $(S 1)_{n}$ \\
\hline STAT2 & nucleus & $(S 2)_{n}$ \\
\hline phosphorylated STAT1 & cytoplasm & $\left(S 1_{p}\right)$ \\
\hline phosphorylated STAT2 & cytoplasm & $\left(S 2_{p}\right)$ \\
\hline homodimer of phosphorylated STAT1 & nucleus & $(S 11)_{n}$ \\
\hline ISGF3 complex & nucleus & $(S 12)_{n}$ \\
\hline inactive X phosphatase & nucleus & $\left(X_{i n}\right)_{n}$ \\
\hline active X phosphatase & nucleus & $\left(X_{a c t}\right)_{n}$ \\
\hline inactive inducer of homodimer degradation & nucleus & $\left(Y_{i n}\right)_{n}$ \\
\hline active inducer of homodimer degradation & nucleus & $\left(Y_{a c t}\right)_{n}$ \\
\hline
\end{tabular}

the original model, that these molecules are activated by the nuclear ISGF3 complex and that their total (i.e., both active and inactive molecules) level is constant:

$$
\begin{aligned}
\frac{\mathrm{d}\left(X_{i n}\right)_{n}}{\mathrm{~d} t}= & -k_{X_{a c t}} \cdot(S 12)_{n} \cdot\left(X_{i n}\right)_{n} \\
& +k_{X_{i n}} \cdot\left(X_{a c t}\right)_{n}, \\
\frac{\mathrm{d}\left(X_{a c t}\right)_{n}}{\mathrm{~d} t}= & k_{X_{a c t}} \cdot(S 12)_{n} \cdot\left(X_{i n}\right)_{n} \\
& -k_{X_{\text {inact }}} \cdot\left(X_{a c t}\right)_{n}, \\
\frac{\mathrm{d}\left(Y_{i n}\right)_{n}}{\mathrm{~d} t}= & -k_{Y_{a c t}} \cdot(S 12)_{n} \cdot\left(Y_{i n}\right)_{n} \\
& +k_{Y_{\text {in }}} \cdot\left(Y_{a c t}\right)_{n}, \\
\frac{\mathrm{d}\left(Y_{a c t}\right)_{n}}{\mathrm{~d} t}= & k_{Y_{a c t}} \cdot(S 12)_{n} \cdot\left(Y_{i n}\right)_{n} \\
& -k_{Y_{\text {inact }}} \cdot\left(Y_{a c t}\right)_{n} .
\end{aligned}
$$

Let us assume that the system described by Eqns. (1)-12 has an equilibrium point, at which

$$
\left.(S 11)_{n}\right|_{0}=0
$$

as suggested by the experimental results. Then, regardless of the mechanism behind the control of the homodimer level and the model parameters, from (8) it stems that the amount of phosphorylated STAT1 in the cytoplasm is also equal to zero, which contradicts the experimental results. This means that the assumption (13) is too strong. Additionally, this shows that models assuming a constant amount of molecules of some type, neglecting their production and degradation (which is an approach often applied in the modeling of signaling pathways) does not allow a more formal analysis, as they will always need the zero equilibrium point (e.g., in the model presented above, if in (1) and (2) there was neither production, nor degradation, it would result in an equilibrium point in which all variables are equal to zero).

Let us now assume that the level of the STAT1 homodimer at the equilibrium is very small, but nonzero. If
$\left(S 1_{p}\right)_{0}$ denotes the value of the cytoplasmic phosphorylated STAT1 protein, this could be written as

$$
\left.(S 11)_{n}\right|_{0}=\left.\alpha\left(S 1_{p}\right)\right|_{0}
$$

where $\alpha$ is a small positive parameter.

Then, if the only regulatory mechanisms were these described in the literature, i.e., only constitutively active phosphatases were taken into account, in (8) the parameters $k_{d x}=0$ and $k_{d y}=0$. This means that from (8)

$$
\left.(S 11)_{n}\right|_{0}=\left.\frac{k_{s 1 s 1}}{k_{\text {inv_s1s1 }}}\left(S 1_{p}\right)^{2}\right|_{0} .
$$

Combining (14) with (15) leads to

$$
k_{i n v_{-} s 1 s 1}=\frac{\left.k_{s 1 s 1} \cdot\left(S 1_{p}\right)\right|_{0}}{\alpha} .
$$

All variables are molar concentrations. Realistic values of the phosphorylated STAT1 protein level should be at least $0.1[\mu M]$ to have the desired effect on gene transcription processes. The value of the parameter $k_{s 1 s 1}$ determining the rate of creating homodimers is of the magnitude $0.1-10$, if the dimers appear in large quantities quickly after the pathway induction. Then, the resulting lower bound of the $k_{i n v_{-} s 1 s 1}$ value would be equal to $0.01 / \alpha$. Even for a relatively large $\alpha$ of 0.1 , which would suggest a relatively large amount of the homodimers at the equilibrium, this translates into the upper bound for the homodimer half-life time of approximately 7 seconds, which is completely unrealistic. This implies the need to include other regulatory processes in the model.

Similarly, the hypothesis about an induced degradation rate, when in (8) the parameters $k_{d x}=0$, would lead to very low levels of both STAT1 and phosphorylated STAT1, not observed in experimental data. In turn, when induced dephosphorylation is applied, with $k_{d x} \neq 0$ and $k_{d x}=0$,

$$
\left.k_{s 1 s 1} \cdot\left(S 1_{p}\right)\right|_{0}=\alpha\left(k_{i n v_{-} s 1 s 1} \cdot+\left.k_{d x} \cdot\left(X_{a c t}\right)\right|_{0}\right),
$$


which can be satisfied for a wide range of biologically acceptable parameters.

Of course, to confirm this hypothesis, appropriate experimental work should take place. However, there are at least several known phosphatases that are responsible for STAT dephosphorylation (Hoeve et al., 2002) and several others are postulated (Yamada et al., 2003). It would be extremely difficult, if possible at all, to conduct experiments in which specific phosphatases would be blocked.

\section{Regulatory mechanisms in late responses}

As mentioned in the preceding sections, the original model of the Interferon- $\beta$ stimulated pathway, presented by Śmieja et al. (2008), provided a good fit to experimental data. However, it failed to explain the source of cytoplasmic IRF1 accumulation, observed in the experiments (7), which took place about 1.5 to 2 hours after the nuclear peak was observed. None of the known negative feedbacks could explain this phenomenon. The behavior of the system has been checked first for varying values of each of the most important parameters (an example is shown in Fig. 97. Following that, a careful analysis of system dynamics was performed for different sets of parameter values in a massive simulation effort. The results obtained suggest that there is an additional, unknown process of negative regulation in the pathway.

Having checked various models, one of them proved to be the most promising. It is known that the IRF1 protein should be activated to act as a TF. This most likely involves phosphorylation by a yet unidentified phosphatase. In the previous models it was assumed that the dynamics of such a process are very fast compared with other processes involved in the pathway and therefore can be neglected. Since the changes introduced there have not yielded desired results, another hypothesis is introduced. It will be assumed that the activation of the process is mediated by a kinase that is constitutively present in the cytoplasm. However, this activation can be blocked by another protein that is produced in the pathway, either by binding the kinase or by promoting its degradation. Contrary to the previous models, the dynamics of IRF1 activation are modeled explicitly. Therefore, it is assumed that the translation process yields an inactive IRF1 protein. The activation of the IRF1 protein in the cytoplasm is blocked by an unknown $\mathrm{X}$ protein and in its absence is a first order process. This protein would be coded by a gene whose transcription is induced in the IFN- $\beta$ activated pathway (Fig. 10). It may be assumed that the Transcription Factor (TF) for this gene should exhibit dynamics similar to those other genes activated in this pathway, so it is not necessary to introduce the variable representing the concentration of such a TF to the model. However, the production of the protein should be delayed with respect to IRF1 dynamics. Such a delay might result from the activation process of the $\mathrm{X}$

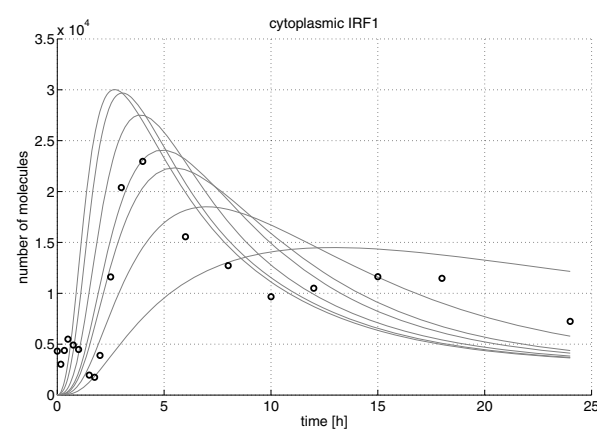

(a)

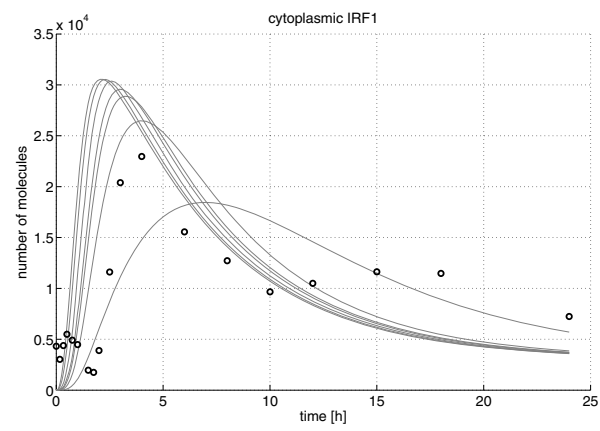

(b)

Fig. 9. Example of different dynamics of a cytoplasmic IRF1 protein when a single parameter was varying: for the original model (a), for the model whose modification is shown in Fig. 10(b). Experimental data are indicated by circles, simulation results for the original model are represented by the dotted line.

protein. In order to avoid introducing too many unknown molecules into the model, it was assumed that the production of the $\mathrm{X}$ protein is similar to that of the IRF1 protein, and its activation is a second-order time-lag process (Śmieja, 2009).

The behavior of the system has been checked first for varying values of each of the most important parameters (an example is shown in Fig. 9). Following that, a careful analysis of system dynamics was performed for different sets of parameter values in a massive simulation effort. The results, presented in Fig. 10 b), suggest that the proposed mechanism can indeed regulate IRF1 cytoplasmic accumulation.

\section{Conclusions}

The paper shows how mathematical modeling can be applied to advance knowledge about regulatory mechanisms in signaling pathways. For early responses, even a rough analysis of equilibrium points can help accept or reject hypotheses about possible regulatory mechanisms. For later responses this is not possible due to the complexity of the 


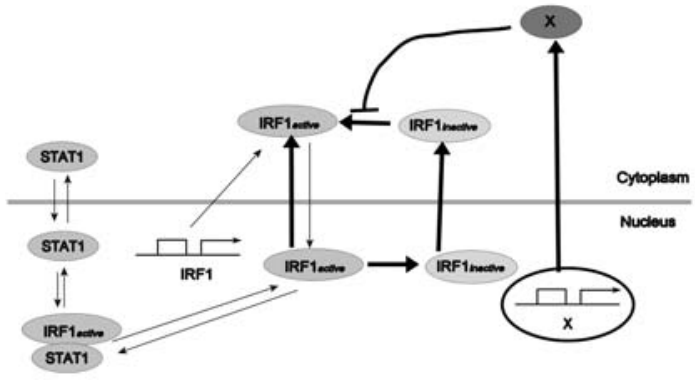

(a)

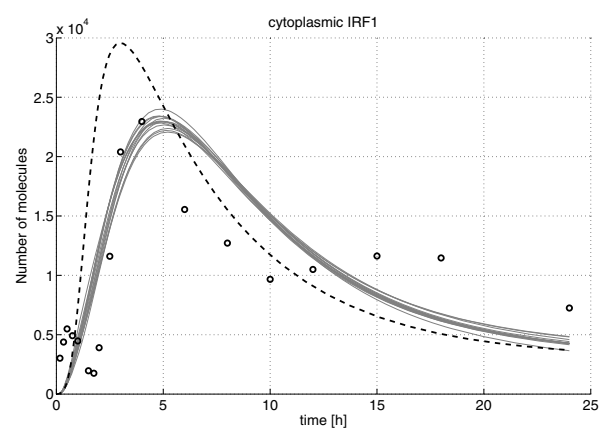

(b)

Fig. 10. Hypothesis about the mechanism controlling the cellular content of the IRF1 protein (a), experimental results (circles), original model (black dashed line) and modified model results for slightly varying parameter sets (grey lines) (b).

models, but then numerical analysis is useful when performed for varying parameters. As a result, the number of experiments necessary to explain the phenomena observed so far and thus build a structural view of the pathway can be reduced.

\section{Acknowledgment}

This work was partially supported by the Polish Ministry for Science and Higher Education under Grant No. N N514 415334 for the years 2007-2010 and was first presented during the 15th National Conference on Application of Mathematics in Biology and Medicine held in Szczyrk, Poland, in 2009.

\section{References}

Alexander, W. and Hilton, D. (2004). The role of suppressors of cytokine signaling (socs) proteins in regulation of the immune response, Annual Review of Immunology 22: 503-529.

Bekisz, J., Schmeisser, H., Hernandez, J., Goldman, N. and Zoon, K. (2004). Human interferons alpha, beta and omega, Growth Factors 22(4): 243-251.

Janeway, C. (2001). Immunobiology 5: The Immune System in Health and Disease, Garland Pub., New York, NY.

Ciliberto, A., Novak, B. and Tyson, J. (2005). Steady states and oscillations in the p53/mdm2 network, Cell Cycle 4: 488-493.

Levy, D.E. and Darnell Jr., J. (2002). Stats: Transcriptional control and biological impact, Nature Reviews Molecular Cell Biology 3: 651-662.

Kalvakolanu, D.V. (2003). Alternate interferon signaling pathways, Pharmacology \& Therapeutics 100: 1-29.

Hoeve, J.D., Ibarra-Sanchez, J., Fu, Y., Zhu, W., Tremblay, M., David, M. and Shuai, K. (2002). Identification of a nuclear stat1 protein tyrosine phosphatase, Molecular and Cellular Biology 22(16): 5662-5668.
Shuai, K. and Liu, B. (2003). Regulation of JAK-STAT signalling in the immune system, Nature Reviews Immunology 3: $900-911$.

Pestka, S., Krause, C. and Walter, M. (2004). Interferons, interferon-like cytokines, and their receptors, Immunological Reviews 202: 8-32.

Sen, G. (2001). Viruses and interferons, Annual Review of Microbiology 55: 255-281.

Śmieja, J. (2009). Advantages and pitfalls of mathematical modelling used for validation of biological hypotheses, Proceedings of the 7th IFAC Symposium on Modelling and Control in Biomedical Systems 2009, Aalborg, Denmark, pp. 348-353.

Śmieja, J., Jamalludin, M., Brasier, A. and Kimmel, M. (2008). Model-based analysis of interferon- $\beta$ induced signaling pathway, Bioinformatics 24(20): 2363-2369.

Lipniacki, T., Paszek, L., Brasier, P., Tian, A., Wang, B., H.-Q., Luxon, B. and Kimmel, M. (2006). Stochastic regulation in early immune response, Biophysical Journal 90: 725-742.

Wormald, S. and Hilton, D. (2004). Inhibitors of cytokine signal transduction, Journal of Biological Chemistry 279(2): 821-824.

Yamada, S., Shiono, S., Joo, A. and Yoshimura, A. (2003). Control mechanism of jak/stat signal transduction pathway, FEBS Letters 534: 190-196.

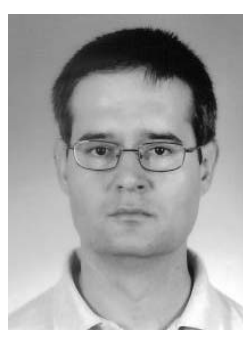

Jaroslaw Śmieja obtained his Ph.D. degree in automatic control and robotics from the Silesian University of Technology, Gliwice, Poland, in 2000. Currently, he is an assistant professor at the Institute of Automatic Control of the same university. His research interests include the application of mathematical modeling and optimization theory in biomedicine as well as systems biology with a particular emphasis on signaling pathways.

Received: 12 February 2010 Revised: 8 June 2010 